\title{
NDRG2 attenuates ischemia-induced astrocyte necroptosis via the repression of RIPK1
}

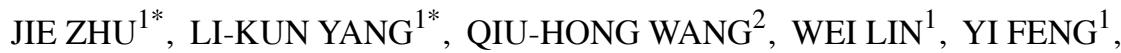 \\ YE-PING XU ${ }^{1}$, WEI-LIANG CHEN ${ }^{1}, \mathrm{KUN} \mathrm{XIONG}^{3}$ and YU-HAI WANG ${ }^{1}$ \\ ${ }^{1}$ Department of Neurosurgery, The 101 Hospital of PLA, School of Medicine, Anhui Medical University, \\ Wuxi, Jiangsu 214044; ${ }^{2}$ Department of Ophthalmology, Wuxi Second Hospital Affiliated to \\ Nanjing Medical University, Wuxi, Jiangsu 214002; ${ }^{3}$ Department of Anatomy and Neurobiology, \\ School of Basic Medical Sciences, Central South University, Changsha, Hunan 410013, P.R. China
}

Received January 18, 2019; Accepted November 20, 2019

DOI: $10.3892 / \mathrm{mmr} .2020 .11421$

\begin{abstract}
Cerebral ischemia results in severe brain damage, and is a leading cause of death and long-term disability. Previous studies have investigated methods to activate astrocytes in order to promote repair in injured brain tissue and inhibit cell death. It has previously been shown that $\mathrm{N}$-myc downstream-regulated gene 2 (NDRG2) was highly expressed in astrocytes and associated with cell activity, but the underlying mechanism is largely unknown. The present study generated NDRG2 conditional knockout (Ndrg2-/-) mice to investigate whether NDRG2 can block ischemia-induced astrocyte necroptosis by suppressing receptor interacting protein kinase 1 (RIPK1) expression. This study investigated astrocyte activity in cerebral ischemia, and identified that ischemic brain injuries could trigger RIP-dependent astrocyte necroptosis. The depletion of NDRG2 was found to accelerate permanent middle cerebral artery occlusion-induced necroptosis in the brain tissue of $\mathrm{Ndrg} 2-/-$ mice, indicating that NDRG2 may act as a neuroprotector during cerebral ischemic injury. The present study suggested that NDRG2 attenuated astrocytic cell death via the suppression of RIPK1. The pharmacological inhibition of astrocyte necroptosis by necrostatin-1 provided neuroprotection against ischemic brain injuries after NDRG2 knockdown. Therefore, NDRG2 could be considered as a potential target for the treatment of cerebral ischemia.
\end{abstract}

Correspondence to: Professor Yu-Hai Wang, Department of Neurosurgery, The 101 Hospital of PLA, School of Medicine, Anhui Medical University, 101 Xingyuan North Road, Wuxi, Jiangsu 214044, P.R. China

E-mail: yuhaiwang@yeah.net

*Contributed equally

Key words: N-myc downstream-regulated gene 2, receptor interacting protein kinase 1, necroptosis, necrostatin-1, astrocyte

\section{Introduction}

Severe craniocerebral trauma is characterized by high mortality and disability, and has been a focus of neuroscientific research (1). Cerebral ischemia is an important pathophysiological feature of secondary brain injury after trauma (2). Secondary injury related mechanisms include primary mechanical injury of blood vessels caused by cerebral tissue ischemia, hematoma and other placeholder lesions caused by local cerebral ischemia (3) In addition, secondary brain injury also includes diffuse brain swelling after trauma, increased intracranial pressure leading to low perfusion in the brain, cerebral vasospasm, microvascular lesions and alterations in local cerebral blood flow, which can influence systemic circulations (3).

Astrocytes are the most widely distributed glial cell type in the central nervous system and play a crucial role in protecting neurons, regulating synaptic function, and promoting nerve regeneration and repair $(4,5)$. Astrocytes also play an important role in the process of ischemic brain injury (6). It was previously shown that astrocytes are regularly distributed in the brain, and establish positional relationships with neurons via close connections to exchange information and material between cells (7). After cerebral ischemia, the extent of the damaged area of brain tissue is determined by the degree of damage to neurons and glial cells (8). The activation of astrocytes can promote neuron damage repair, axon regeneration and neuron migration (9). Previous studies have shown that astrocytes maintain the homeostasis of the extracellular microenvironment of brain tissue, and can promote neuron survival after cerebral ischemia by interacting with neurons and glial cells, and participating in the process of endogenous nerve protection $(10,11)$. Therefore, understanding how to activate the neuroprotective mechanisms of astrocytes and inhibit cell death could facilitate the treatment of ischemia-induced brain tissue injury.

Necroptosis is a programmed type of necrosis, which can be regulated and reversed under certain conditions $(12,13)$. Necroptosis is mediated by the interaction between receptor interacting protein (RIP) kinase (RIPK) members, such as RIPK1 and RIPK3 $(12,13)$. After external signal stimulation, a 
series of death receptors are activated, such as tumor necrosis factor receptor (TNFR)1, TNFR2 and Fas ligand (14). RIPK1 is then activated and interacts with death receptors to form complex-I, which can recruit RIPK3 to form a necrosome, which is critical for the process of necroptosis (14). A previous study showed that the cerebral ischemic environment could lead to necroptosis of neurons and astrocytes, and increase the area of brain injury (15). Based on the importance of astrocytes in the repair of injured brain tissue, inhibiting astrocytic death and promoting its normal activation is key to the functional recovery of the nervous system, as well as the treatment and prognosis of traumatic cerebral ischemia.

N-myc downstream-regulated gene 2 (NDRG2) belongs to the NDRG family that consists of four identified members (NDRG1-4), and has been implicated in the regulation of cell differentiation and proliferation $(16,17)$. NDRG2 is closely related to neurological diseases (18). In the brains of patients with Alzheimer's disease, NDRG2 expression is upregulated $(18,19)$. Overexpression of NDRG2 in nerve growth factor induced-differentiated PC12 cells promotes axon regeneration (19). In astrocytes, NDRG2 is specifically and more widely expressed than glial fibrillary acidic protein (GFAP), which is a commonly used astrocyte marker; thus, NDRG2 is considered as a new astrocyte marker (20). It was previously shown that NDRG2 could regulate astrocyte activity by promoting cell differentiation and stabilizing cell morphology (21). The present study generated NDRG2 conditional knockout mice (Ndrg2-/-) to investigate the association of NDRG2 and astrocytic activity during cerebral ischemia.

\section{Materials and methods}

Animals. NDRG2 conditional knockout (Ndrg2-/-) mice were generated via cooperation with Shanghai Biomodel Organism Science \& Technology Development Co., Ltd. and maintained on C57BL6/J background as previously described (22). Briefly, $\mathrm{Ndrg} 2^{\text {flox/flox }}$ mice were crossed with B6.C-Tg(CMV-cre) $1 \mathrm{Cgn} / \mathrm{J}$ mice (Jackson Laboratory) to generate Ndrg2-/- mice. The line was backcrossed with C57BL/6J a minimum of 8 times before use in any experiments in this study. Heterozygous mice carrying the knockout mutation were interbred to obtain the homozygous Ndrg2-deficient mice and their wild-type (WT) littermates. All animals were raised under specific pathogenfree conditions. The incubator temperature was maintained at $21 \pm 2^{\circ} \mathrm{C}$ with $45-60 \%$ humidity, on a $12 / 12 \mathrm{~h}$ day/night cycle with food and water ad libitum. WT mice and $\mathrm{Ndrg} 2-/-$ mice were age-matched, and all mice used were male. In total, 60 mice were used in this study and 8 mice were used for each group of treatment, all experiments were repeated $\geq 3$ times. All animal experiments were in accordance with the relevant provisions of the Laboratory Animal Center and were approved by the Experimental Animal Ethics Committee of the 101 Hospital of PLA of Anhui Medical University.

Permanent middle cerebral artery (MCA) occlusion (pMCAO). pMCAO was performed as previously described (23). For all experiments, male C57BL/6 mice (age, 7 weeks; weight, 18-22 g) were used. Following anesthesia with $2.5 \%$ isoflurane in $100 \% \mathrm{O}_{2}$, holes were drilled to place the laser. A nylon filament was advanced from the right common carotid artery through the internal carotid to the MCA. Rectal temperature was maintained at $37.0 \pm 0.5^{\circ} \mathrm{C}$ using a temperature-regulated heating pad during the procedure. Animals were randomly assigned to different treatment groups ( $n=8 /$ group). Infarct volume was assessed with a direct method using ImageJ v2.1 (National Institutes of Health). The grip forces of the mice were measured as previously described (24).

Primary cortical astrocyte culture and NDRG2 lentivirus infection. The 1-or 2-day-old neonates $(n=12)$ were used for primary cortical astrocyte culture as previously described (25). The cerebral cortexes were digested with $0.25 \%$ trypsin for $10 \mathrm{~min}$ at $37^{\circ} \mathrm{C}$ and filtered with a sterile nylon cell strainer. The cells were grown at $37^{\circ} \mathrm{C}$ in conditions of $5 \% \mathrm{CO}_{2}$ and 95\% $\mathrm{O}_{2}$ in DMEM/F12 (1:1) supplemented with 10\% FBS (both purchased from Gibco; Thermo Fisher Scientific, Inc.), streptomycin $(100 \mu \mathrm{g} / \mathrm{ml})$, and penicillin $(100 \mathrm{U} / \mathrm{ml})$. For NDRG2 overexpression in astrocytes, cells were infected with $1 \times 10^{8} \mathrm{TU} / \mathrm{ml}$ NDRG2-overexpressing lentivirus (pHBLV-CMVIE-NDRG2), which was packaged by Hanbio Biotechnology Co., Ltd., with $5 \mu \mathrm{g} / \mathrm{ml}$ polybrene. At $48 \mathrm{~h}$ after infection, subsequent experiments were performed.

Oxygen-glucose deprivation (OGD) treatment. OGD was performed as previously described (26). Cells in the OGD group were cultured in glucose-free DMEM (Gibco; Thermo Fisher Scientific, Inc.) and kept for $6 \mathrm{~h}$ or $12 \mathrm{~h}$ at $37^{\circ} \mathrm{C}$ in a hypoxic incubator chamber (Billups-Rothenberg, Inc.) filled with $95 \% \mathrm{~N}_{2} / 5 \% \mathrm{CO}_{2}$. Cells grown in conditions of $5 \% \mathrm{CO}_{2}$ and $95 \% \mathrm{O}_{2}$ in standard DMEM were used as the control group. Necrostatin-1 (Nec-1; $10 \mu \mathrm{M}$; Sigma-Aldrich; Merck $\mathrm{KGaA}$ ) dissolved in DMSO was added to the cells $0.5 \mathrm{~h}$ before OGD treatment.

Western blotting. Cells were lysed in RIPA buffer (Thermo Fisher Scientific, Inc.) and the concentrations were measured by bicinchoninic acid protein assay kit (Thermo Fisher Scientific, Inc.). In all samples, $40 \mu \mathrm{g}$ proteins were separated by $12 \%$ SDS-PAGE and transferred to nitrocellulose membranes (Hybond-ECL; GE Healthcare Life Sciences). Then, nitrocellulose membranes were incubated with 5\% skimmed milk for $1 \mathrm{~h}$ at room temperature. Primary antibodies were then introduced at dilutions of 1:1,000 for anti-RIPK1 (cat. no. ab106393; Abcam), anti-GFAP (cat. no. AB5541; EMD Millipore), anti-NDRG2 (cat. no. 5667; Cell Signaling Technology, Inc.) and anti- $\beta$-actin (cat. no. 4970; Cell Signaling Technology, Inc.), and membranes were incubated at $4^{\circ} \mathrm{C}$ overnight. The following secondary antibodies $(1: 5,000)$ were then added against the primary antibodies, horseradish peroxidase (HRP)-conjugated anti-mouse IgG (cat. no. 7076; Cell Signaling Technology, Inc.) or HRP-conjugated antirabbit IgG (cat. no. 7074; Cell Signaling Technology, Inc.), and membranes were incubated for $1 \mathrm{~h}$ at room temperature. The blots were detected by chemiluminescence (Pierce; Thermo Fisher Scientific, Inc.) or Odyssey Imaging System (LI-COR Biosciences).

Immunofluorescence assay. The brain tissue was fixed with $10 \%$ formalin overnight at $4^{\circ} \mathrm{C}$ and embedded in paraffin. The sections (thickness, $6 \mu \mathrm{m}$ ) were then dewaxed in xylene 
A

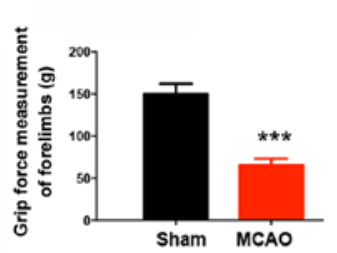

E

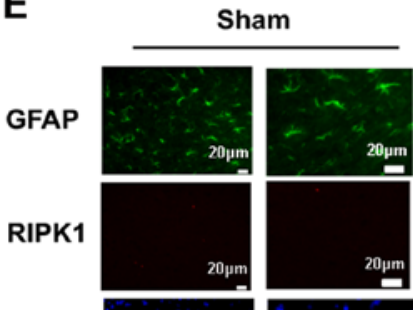

DAPI
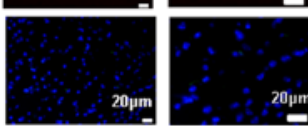

Merge
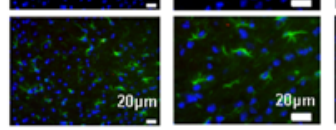

J

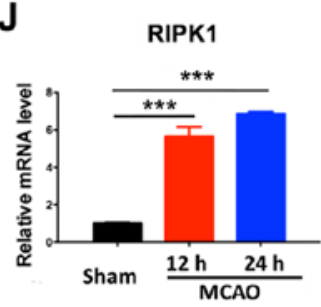

\section{L}

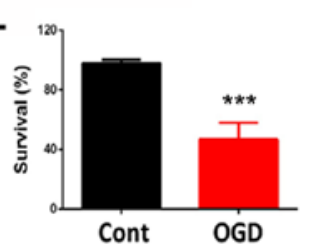

B
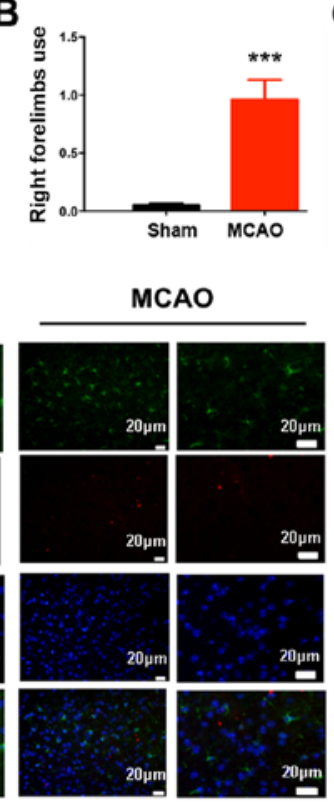

K

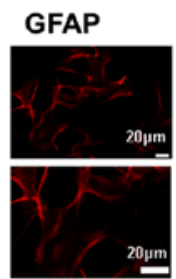

M

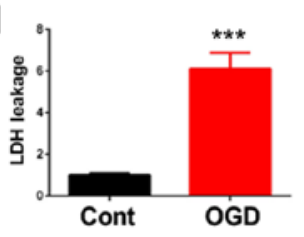

C

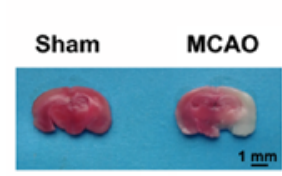

$\mathbf{F}$

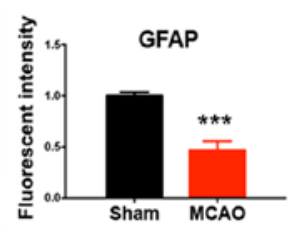

H

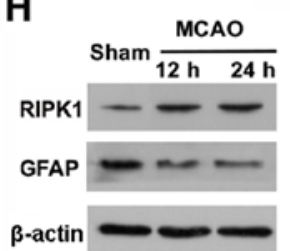

NeuN

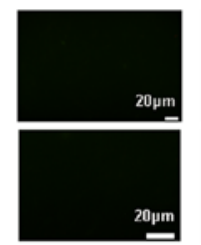

DAP

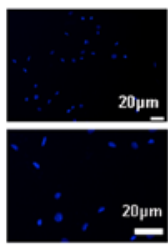

N

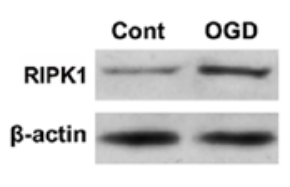

D

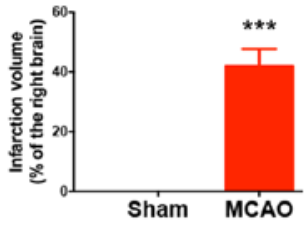

G

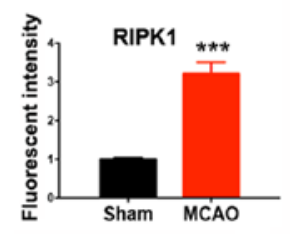

I

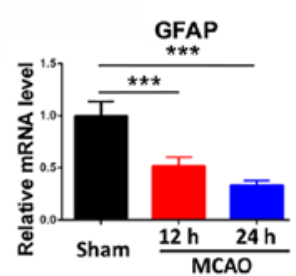

Merge

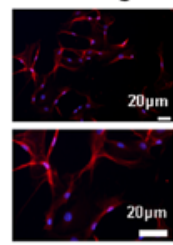

$\mathbf{O}$

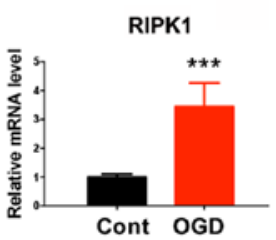

Figure 1. Ischemic brain injuries trigger RIP-dependent astrocyte necroptosis. In a pMCAO mouse model, $24 \mathrm{~h}$ after ischemia, the (A) grip force, (B) right forelimb use and (C and D) infarction volume were determined. Mean $\pm \mathrm{SD} ; \mathrm{n}=8$ /group. (E) Immunofluorescent staining in the mouse brain tissues was used to quantify (F) GFAP and (G) RIPK1 expression levels. (H) Western blotting results for GFAP and RIPK1 protein expression levels at indicated times after the pMCAO operation. (I) GFAP and (J) RIPK1 mRNA expression levels were measured by reverse transcription-quantitative PCR at indicated times after the pMCAO operation. ${ }^{* * *} \mathrm{P}<0.001$ vs. Sham. (K) Primary cortical astrocytes were stained with GFAP and NeuN to determine the purity. (L) Cell survival and (M) LDH leakage of astrocytes were determined $12 \mathrm{~h}$ after OGD treatment. (N) RIPK1 protein and (O) mRNA expression levels were determined $6 \mathrm{~h}$ after OGD treatment. ${ }^{* * *} \mathrm{P}<0.001$ vs. Control. RIP, receptor interacting protein; RIPK1, receptor interacting protein kinase 1; pMCAO, permanent middle cerebral artery occlusion; GFAP, glial fibrillary acidic protein; LDH, lactate dehydrogenase; NeuN, neuronal nuclei; OGD, oxygen-glucose deprivation.

three times for $5 \mathrm{~min}$ each at room temperature and rehydrated in a descending series of ethanol (100 and 95\%) at room temperature for $10 \mathrm{~min}$ each. For the cells, the primary cells were fixed in $4 \%$ paraformaldehyde for $10 \mathrm{~min}$ at room temperature. For immunofluorescent staining, the sections were blocked with 5\% normal goat serum (cat. no. 5425; Cell Signaling Technology, Inc.) and 0.3\% Triton X-100 for $1 \mathrm{~h}$ at room temperature to minimize non-specific staining. They were then incubated overnight at $4^{\circ} \mathrm{C}$ with the following primary antibodies (1:200), anti-RIPK1 (cat. no. ab106393; Abcam), anti-GFAP (cat. no. AB5541; EMD Millipore) and anti-NeuN (cat. no. 24307; Cell Signaling Technology, Inc.). Then, the samples were incubated with HRP-conjugated secondary antibodies (1:500) for $1 \mathrm{~h}$ at room temperature (cat. nos. A32935, A32731 and A32759; Thermo Fisher Scientific, Inc.), and further incubated with $10 \mu \mathrm{g} / \mathrm{ml}$ DAPI for $5 \mathrm{~min}$ at room temperature for visualization of the cell nuclei. The cells were imaged by confocal laser scanning microscopy (magnification, x1,000; Nikon Corporation) and the fluorescence intensity of immunofluorescence images was measured by ImageJ v2.1 (National Institutes of Health) for quantification of five fields per sample.

Reverse transcription-quantitative PCR (RT-qPCR). Total RNA was isolated from brain tissue or primary cells using TRIzol $^{\circledR}$ reagent (Invitrogen; Thermo Fisher Scientific, Inc.) and then quantified. Then, $2 \mu \mathrm{g}$ of total RNA was reverse-transcribed using reverse transcriptase (Promega 
A

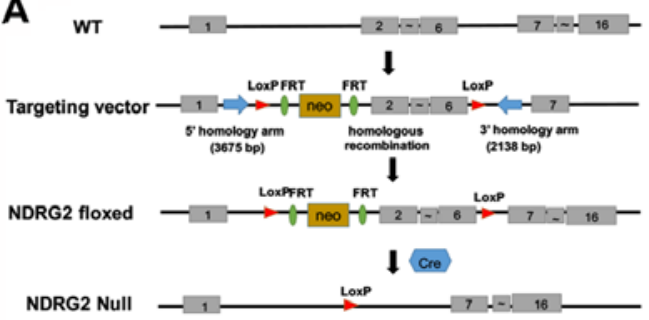

D

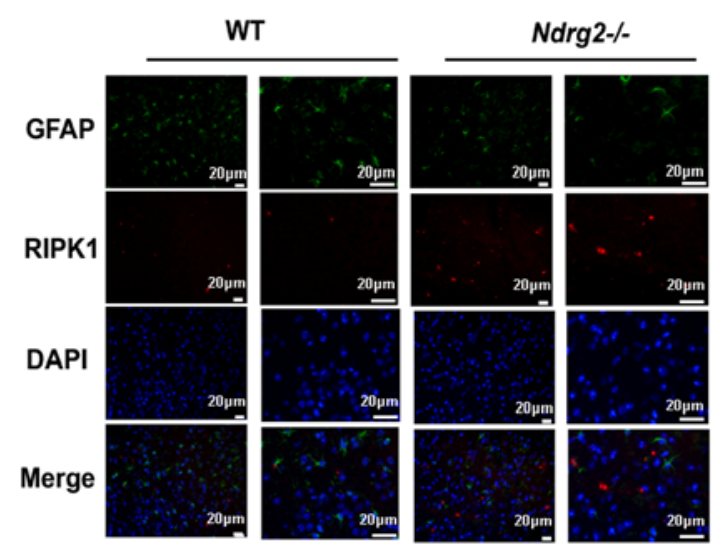

B

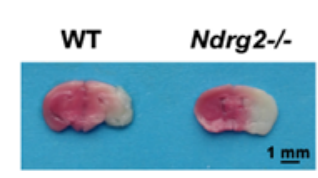

E

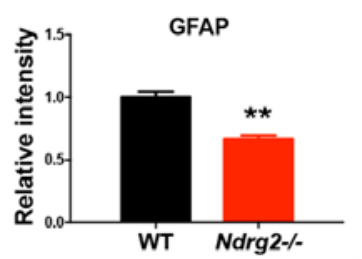

C

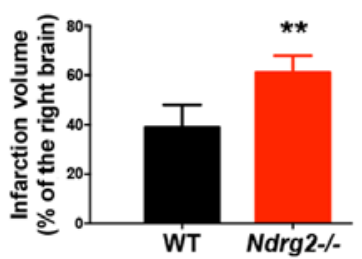

F

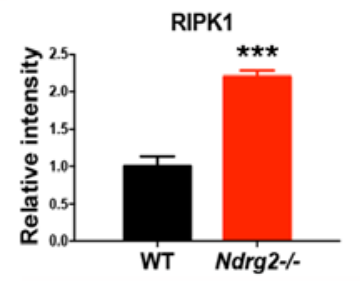

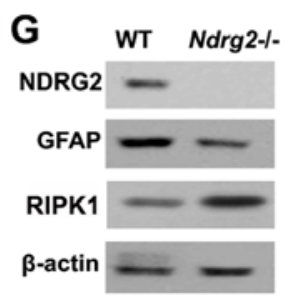
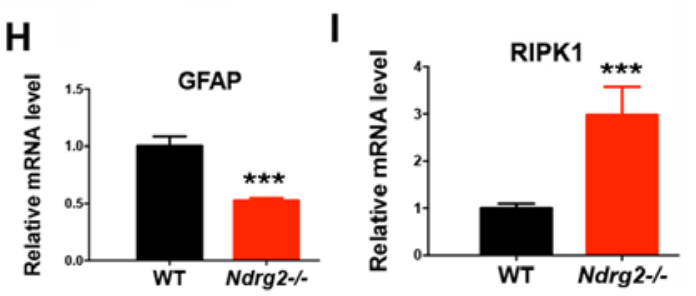

Figure 2. NDRG2 depletion accelerates cerebral ischemic injury-induced necroptosis. (A) Schematic representation of the NDRG2 targeting vector: First, WT locus; second, targeted allele; third, $N d r g 2$-floxed; fourth, null allele $N d r g 2$-Null. Exons are represented by solid rectangles. The targeting vector, which is flanked by 3,675 bp of 5' homology and 2,138 bp of 3' homology, contains a LoxP-FRT-Neo-FRT cassette and the second LoxP sequence in introns 1 and 6 , respectively. WT or Ndrg2-/- mice received pMCAO operation. (B) At $24 \mathrm{~h}$ following pMCAO surgery, the infarction volume was determined and (C) quantified. At $12 \mathrm{~h}$ following pMCAO surgery, the indicated proteins levels were determined by (D-F) immunofluorescent staining and (G) western blotting, and mRNA expression levels of (H) GFAP and (I) RIPK1 were determined by reverse transcription-quantitative PCR. ${ }^{* *} \mathrm{P}<0.01,{ }^{* * * *} \mathrm{P}<0.001$ vs. WT group. NDRG2, $\mathrm{N}$-myc downstream-regulated gene 2; WT, wild-type; RIPK1, receptor interacting protein kinase 1; pMCAO, permanent middle cerebral artery occlusion; GFAP, glial fibrillary acidic protein; LoxP, locus of X-over P1.

Corporation) according to the manufacturer's instructions. qPCR was performed with TB Green ${ }^{\circledR}$ Fast qPCR kit (Takara Bio, Inc.) for 35 cycles of: $10 \mathrm{sec}$ at $98^{\circ} \mathrm{C}, 10 \mathrm{sec}$ at $55^{\circ} \mathrm{C}$ and $20 \mathrm{sec}$ at $72^{\circ} \mathrm{C}$. The primer sequences used were as follows: GFAP, forward 5'-CGGAGACGCATCACCTCTG-3' and reverse 5'-TGGAGGAGTCATTCGAGACAA-3'; RIPK, forward 5'-GACAGACCTAGACAGCGGAG -3' and reverse 5'-CCAGTAGCTTCACCACTCGAC-3'; GAPDH, forward 5'-AGGTCGGTGTGAACGGATTTG-3' and reverse 5'-GGGGTCGTTGATGGCAACA-3'. The mean Cq values for the target genes were normalized to the mean $\mathrm{Cq}$ value for the endogenous control GAPDH as previously described (27). The ratio of mRNA expression of target gene to GAPDH was defined as $2^{-\Delta \Delta C q}$.

Cell growth assay. For the crystal violet assays, $1 \times 10^{5}$ astrocytes were seeded into each well of a 12-well plate with three replicates for each group. Cells in conditions of $5 \% \mathrm{CO}_{2}$ and 95\% $\mathrm{O}_{2}$ in DMEM/F12 supplemented with $10 \%$ FBS were used as a negative control. After $12 \mathrm{~h}$ of OGD treatment, cell proliferation was assessed by crystal violet staining at $37^{\circ} \mathrm{C}$ for $4 \mathrm{~h}$, as described previously (28). For the cell survival assays, $1 \times 10^{4}$ cells from each treatment condition were plated in triplicate in a 96 -well plate at $37^{\circ} \mathrm{C}$. The survival rate was measured using a Guava Nexin assay (EMD Millipore). Results are shown as the mean $\%$ of viable cells.
Lactate dehydrogenase ( $L D H)$ assay. A total of $1 \times 10^{5}$ astrocytes were seeded into each well of a 6-well plate. The release of LDH was measured using the LDH assay kit (cat. no. A020-2-2; Nanjing Jiancheng Bioengineering Institute Co., Ltd.) according to the manufacturer's protocols. Samples without coenzyme I treatment were used as a negative control. LDH leakage was calculated as follows: LDH leakage $(\%)=$ (A positive/A positive blank) / (A negative/A negative blank) $\mathrm{x} 100 \%$.

Statistical analysis. Data are presented as the mean \pm SD. Statistical analysis was conducted using SPSS 10.0 software (SPSS, Inc.) to perform ANOVA followed by a StudentNewman-Keuls post hoc test. $\mathrm{P}<0.05$ was considered to indicate a statistically significant difference.

\section{Results}

Ischemic brain injuries trigger RIP-dependent astrocyte necroptosis. To determine the association of cerebral ischemic injury and astrocytic features, a brain injury mouse pMCAO model was established. The present results showed decreased grip strength and an increased rate of right forelimb utilization in pMCAO mice (Fig. 1A and B). In addition, a significant infarct was found in the ipsilateral cortex of pMCAO brains compared with sham-operated brains (Fig. 1C and D), 

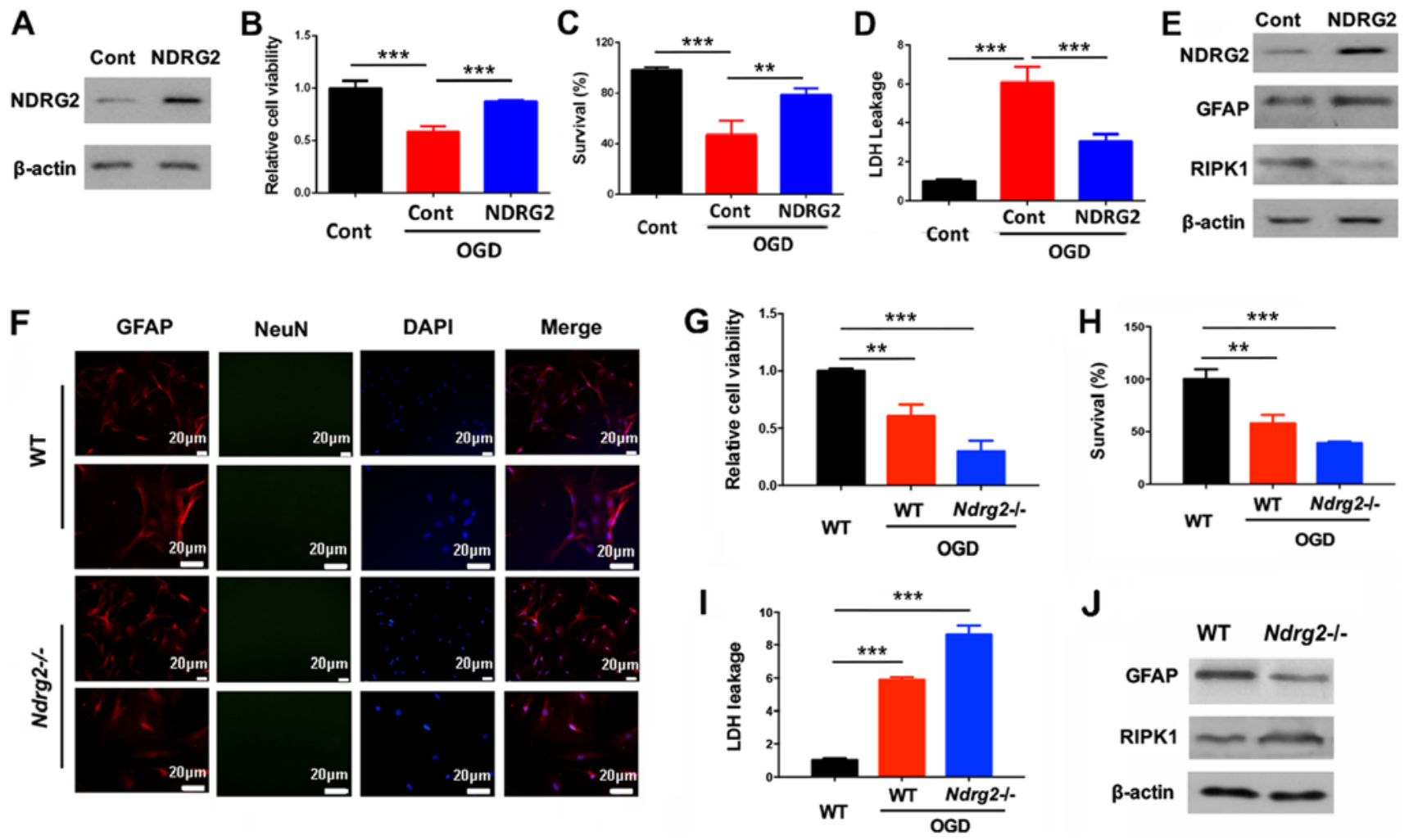

Figure 3. NDRG2 attenuates astrocytic cell death via the suppression of RIPK1. Astrocytes were infected with NDRG2 overexpressing lentivirus. (A) NDRG2 expression was determined after the lentivirus infection. (B) Cell viability, (C) survival and (D) LDH leakage were determined $12 \mathrm{~h}$ after OGD treatment. (E) Protein expression levels of GFAP and RIPK1 were determined $6 \mathrm{~h}$ after OGD treatment. (F) Primary cortical astrocytes were stained with GFAP and NeuN to determine the purity. Astrocytes were isolated from WT or Ndrg2-/- mice. (G) Cell viability, (H) survival and (I) LDH leakage were determined $12 \mathrm{~h}$ after OGD treatment. (J) Protein levels were determined $6 \mathrm{~h}$ after OGD treatment. ${ }^{* *} \mathrm{P}<0.01,{ }^{* * *} \mathrm{P}<0.001$. NDRG2, N-myc downstream-regulated gene 2; WT, wild-type; RIPK1, receptor interacting protein kinase 1; GFAP, glial fibrillary acidic protein; LDH, lactate dehydrogenase; NeuN, neuronal nuclei; OGD, oxygen-glucose deprivation.

indicating that the pMCAO mouse model was successfully established. The present results showed that protein and mRNA expression levels of GFAP were decreased at 12 and $24 \mathrm{~h}$ after pMCAO in the ischemic cortex (Fig. 1E, F, H and I). The protein and mRNA expression levels of RIPK1, an initiator of necroptosis, were increased in the $\mathrm{pMCAO}$ group compared with the control (Fig. 1E, G, H and J). Therefore, the present results suggested that ischemic brain injuries could be associated with RIP-dependent necroptosis. Protein and mRNA expression levels of RIPK1 were also increased in astrocytes after OGD exposure, in addition to decreased cell survival rate and increased LDH leakage (Fig. 1K-O).

NDRG2 knockdown accelerates cerebral ischemic injuryinduced necroptosis. Previous studies have demonstrated that the tumor suppressor NDRG2 has a neuroprotective function in various diseases $(29,30)$. Therefore, the present study investigated whether NDRG2 was involved in the regulation of cerebral ischemic injury and necroptosis. A mouse line carrying the NDRG2 conditional knockout allele (Ndrg2-/-) with exons 2-6 flanked by locus of X-over P1 sites (Fig. 2A) was generated. Female homozygotes ( $\mathrm{Ndrg2-/-)}$ were crossed with CMV-Cre transgenic male mice, which have ubiquitous Cre activity. After the pMCAO operation, an increased infarction volume was observed in Ndrg2-/- mice (Fig. 2B and C); thus, NDRG2 may provide neuroprotection against pMCAO insult. Moreover, NDRG2 knockout led to a significant decrease in GFAP expression levels, and a significant increase in the protein and mRNA expression levels of RIPK1 (Fig. 2D-I). Therefore, the depletion of NDRG 2 could accelerate pMCAOinduced necroptosis in brain tissue. NDRG2 may function as a neuroprotectant during cerebral ischemic injury.

NDRG2 attenuates astrocytic cell death via the suppression of RIPK1. To further determine the role of NDRG2 in regulating astrocyte survival, astrocytes from the neonate mice were isolated and NDRG2 was overexpressed using a lentivirus (Fig. 3A). After OGD exposure, NDRG2 overexpression could significantly block OGD-dependent decreases in cell viability and survival, and induction of LDH leakage (Fig. 3B-D). Furthermore, NDRG2 overexpression increased GFAP expression levels and decreased RIPK1 expression levels after OGD exposure (Fig. 3E), indicating NDRG2 may attenuate astrocytic cell death by repressing RIPK1 levels. In addition, astrocytes were also isolated from wild-type and $\mathrm{Ndrg} 2-/-$ mice (Fig. 3F). NDRG2 knockout increased OGD-induced cell death and LDH leakage (Fig. 3G-I). NDRG2 knockout also upregulated RIPK1 and downregulated under OGD conditions GFAP (Fig. 3J). Therefore, the present results suggested that NDRG2 could block OGD-induced astrocytic cell death by suppressing RIPK1.

Necroptosis inhibitor necrostatin-1 blocks astrocytic cell death after NDRG2 knockdown. In order to determine 

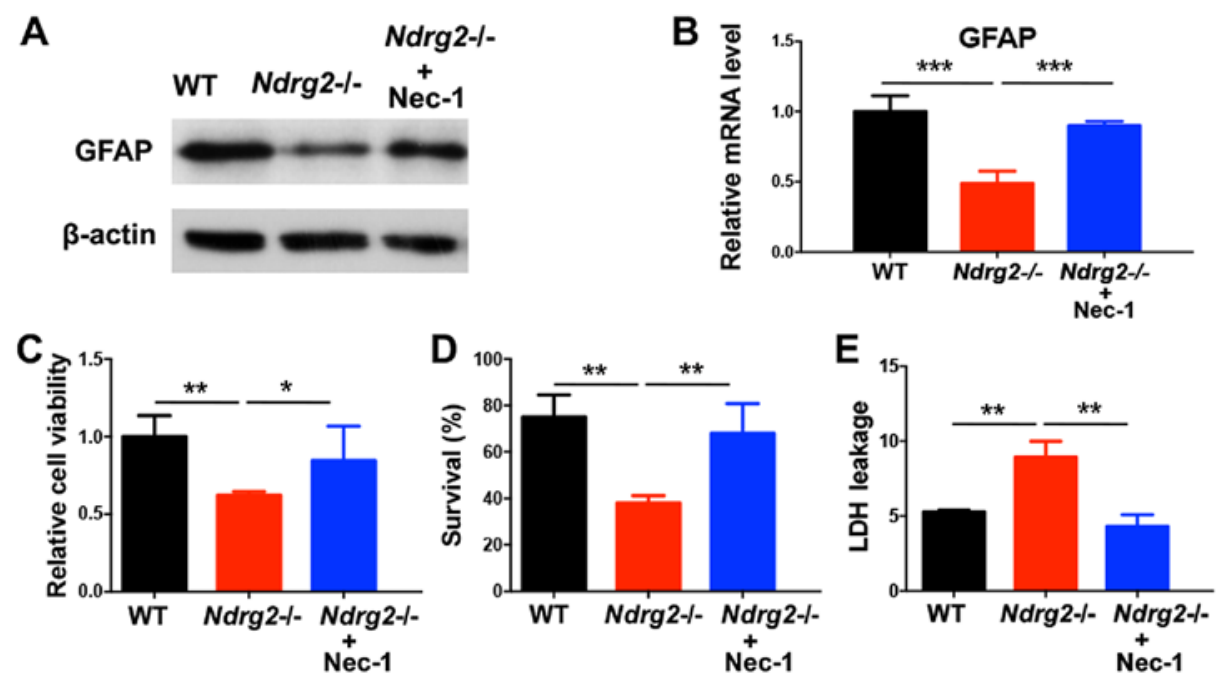

Figure 4. Necroptosis inhibitor Nec-1 blocks astrocytic cell death after NDRG2 knockdown. Nec-1 $(10 \mu \mathrm{M})$ was administrated to primary cultured astrocytes for $30 \mathrm{~min}$ prior to OGD. (A) GFAP protein and (B) mRNA expression levels were determined $6 \mathrm{~h}$ after OGD treatment. (C) Cell viability, (D) survival and (E) LDH leakage were determined $12 \mathrm{~h}$ after OGD treatment. ${ }^{*} \mathrm{P}<0.05,{ }^{* * *} \mathrm{P}<0.01,{ }^{* * * *} \mathrm{P}<0.001$. Nec-1, necrostatin-1; NDRG2, N-myc downstream-regulated gene 2; WT, wild-type; RIPK1, receptor interacting protein kinase 1; GFAP, glial fibrillary acidic protein; LDH, lactate dehydrogenase; OGD, oxygen-glucose deprivation.

whether the role of NDRG2 in neuroprotection is the result of blocking necroptosis, the present study used a necroptosis inhibitor Nec-1, which can inhibit necrosome formation via the suppression of RIPK1 activity (31). Nec-1 treatment prevented the pMCAO-induced decrease in GFAP protein and mRNA expression levels in the ischemic cortex of $\mathrm{Ndrg} 2-/-$ mice (Fig. 4A and B). In addition, after Nec-1 treatment, the reduced viability and survival rates of astrocytes from $\mathrm{Ndrg} 2-/-$ mice following OGD exposure were partly reversed (Fig. 4C and D). OGD-induced LDH leakage from $\mathrm{Ndrg} 2-/$ - astrocytes was also attenuated following Nec-1 treatment (Fig. 4E). The present results indicated that NDRG2 could function as a neuroprotector by blocking necroptosis, and that pharmacological inhibition of astrocyte necroptosis promotes neuroprotection against ischemic brain injuries after NDRG2 knockdown.

\section{Discussion}

Cerebral ischemia is an important component of secondary brain injury after trauma (2). Inhibiting ischemic injury has become one of the key therapeutic methods for improving the prognosis of brain injury (3). A highly regulated form of necrosis, termed necroptosis, elicited significant interest in the study of human diseases such as ischemic stroke in order to understand its implications in pathologies (32). The present study investigated astrocyte activity during cerebral ischemia, and identified that ischemic brain injuries may trigger RIP-dependent astrocyte necroptosis. In a NDRG2 conditional knockout mouse model, the present study found that knockdown of NDRG2 could accelerate pMCAO-induced necroptosis in brain tissue, therefore indicating that NDRG2 may function as a neuroprotector during cerebral ischemic injury. The present study suggested that NDRG2 may attenuate astrocytic cell death via the suppression of RIPK1. The present results suggested that pharmacological inhibition of astrocyte necroptosis by Nec-1 produced neuroprotection against ischemic brain injuries after NDRG2 knockdown. Therefore,
NDRG2 could be considered as a target for the treatment of cerebral ischemia.

Astrocytes are the most widely distributed glial cells in the nervous system $(4,5)$. Astrocytes can not only provide metabolic and nutritional support for neurons, but also play a crucial role in promoting neuronal survival, synaptic function, nerve regeneration and nerve repair $(4,5)$. It is of clinical importance to explore the mechanism of astrocyte death to facilitate the treatment and prognosis of post-traumatic cerebral ischemia. To determine the association between cerebral ischemic injury and astrocytes, the present study established a brain injury mouse pMCAO model and identified that RIPK1, which is an initiator of necroptosis, was upregulated after injury. OGD exposure also led to increased RIPK1 expression levels and LDH leakage, and decreased cell survival rate. Therefore, the present results suggested that ischemic brain injuries may be associated with RIP-dependent astrocyte necroptosis.

NDRG2 has been shown to function as a tumor suppressor via the reduction of cell proliferation and metastasis, and induction of cell differentiation in numerous cancer types $(33,34)$. In the central nervous system, NDRG2 is highly expressed in astrocytes, and regulates astrocytic activity by promoting cell differentiation and stabilizing cell morphology (21). To investigate the role of NDRG2 in astrocytes, the present study generated $\mathrm{Ndrg} 2-/$ - mice. After ischemic injury, increased infarction volume, reduction of GFAP and induction of RIPK1 were observed; thus, NDRG2 may provide neuroprotection against cerebral ischemia. Moreover, astrocytes from $\mathrm{Ndrg} 2-/$ - mice showed decreased cell viability and survival, and increased LDH leakage after OGD exposure. The present study suggested that NDRG2 could block OGD-induced astrocytic cell death by inhibiting RIPK1. A similar conclusion was reached following NDRG2 overexpression in astrocytes. Therefore, the present study suggested that NDRG2 plays an important role in blocking cerebral ischemic injury-induced necroptosis, potentially via the suppression of RIPK1 expression. 
Necroptosis is different to traditional apoptosis. When cells are stimulated by tumor necrosis factor- $\alpha$ and other apoptotic inducers, necrotic morphological features can be induced, and treatment with caspase inhibitors can block apoptosis pathways (35). Nec-1 is one of the most commonly used RIPK1 inhibitors. The effect of Nec-1 on necroptosis is associated with allosteric inhibition of the activity of RIPK1 by interacting with the T-loop of the N-terminal kinase domain (36). A previous study showed that Nec-1 can decrease ischemic brain injury area dose-dependently, and improve nerve function score, prolong nerve protective effect and delay the onset of necroptosis by inhibiting the activation of RIPK1/RIPK3 pathways after ischemic brain injury (19). In addition, Nec-1 can protect neurovasculature and improve the prognosis of animals following neurological injury $(37,38)$. The present results indicated that Nec-1 treatment prevented pMCAOinduced reductions of GFAP, ODG-induced suppression of astrocyte viability and survival, and induction of LDH leakage in the ischemic cortex of Ndrg2-/- mice. Therefore, pharmacological inhibition of astrocyte necroptosis may provide neuroprotection against ischemic brain injuries after NDRG2 knockdown. NDRG2 could be considered as a target for the treatment of cerebral ischemia.

\section{Acknowledgements}

Not applicable.

\section{Funding}

This work was financially supported by the National Natural Science Foundation of China (grant no. 81601719).

\section{Availability of data and materials}

The datasets used and/or analyzed during the current study are available from the corresponding author on reasonable request.

\section{Authors' contributions}

YHW and KX contributed to conception and design of this study. JZ, LKY, QHW, WL and YF performed the experiments. YPX and WLC contributed to the analysis and interpretation of data. All authors read and approved the final manuscript.

\section{Ethics approval and consent to participate}

All animal experiments were in accordance with the relevant provisions of the Laboratory Animal Center and were approved by the Experimental Animal Ethics Committee of the 101 Hospital of PLA of Anhui Medical University.

\section{Patient consent to participate}

Not applicable.

\section{Competing interests}

The authors declare that they have no competing interests.

\section{References}

1. Hyder AA, Wunderlich CA, Puvanachandra P, Gururaj G and Kobusingye OC: The impact of traumatic brain injuries: A global perspective. NeuroRehabilitation 22: 341-353, 2007.

2. Bouma GJ, Muizelaar JP, Choi SC, Newlon PG and Young HF: Cerebral circulation and metabolism after severe traumatic brain injury: The elusive role of ischemia. J Neurosurg 75: 685-693, 1991.

3. Piper LC, Zogg CK, Schneider EB, Orman JA, Rasmussen TE, Blackbourne LH and Haider AH: Guidelines for the Treatment of Severe Traumatic Brain Injury: Are They Used? JAMA Surg 150: 1013-1015, 2015.

4. Davila D, Thibault K, Fiacco TA and Agulhon C: Recent molecular approaches to understanding astrocyte function in vivo. Front Cell Neurosci 7: 272, 2013.

5. Ota Y, Zanetti AT and Hallock RM: The role of astrocytes in the regulation of synaptic plasticity and memory formation. Neural Plast 2013: 185463, 2013

6. Bambrick L, Kristian T and Fiskum G: Astrocyte mitochondrial mechanisms of ischemic brain injury and neuroprotection. Neurochem Res 29: 601-608, 2004.

7. Trendelenburg G and Dirnagl U: Neuroprotective role of astrocytes in cerebral ischemia: Focus on ischemic preconditioning. Glia 50: 307-320, 2005.

8. Nakashima MN, Yamashita K, Kataoka Y, Yamashita YS and Niwa M: Time course of nitric oxide synthase activity in neuronal, glial, and endothelial cells of rat striatum following focal cerebral ischemia. Cell Mol Neurobiol 15: 341-349, 1995.

9. Anderson MF, Blomstrand F, Blomstrand C, Eriksson PS and Nilsson M: Astrocytes and stroke: Networking for survival? Neurochem Res 28: 293-305, 2003.

10. Dienel GA and Hertz L: Astrocytic contributions to bioenergetics of cerebral ischemia. Glia 50: 362-388, 2005.

11. Swanson RA, Ying W and Kauppinen TM: Astrocyte influences on ischemic neuronal death. Curr Mol Med 4: 193-205, 2004.

12. Kim SJ and Li J: Caspase blockade induces RIP3-mediated programmed necrosis in Toll-like receptor-activated microglia. Cell Death Dis 4: e716, 2013.

13. Shindo R, Kakehashi H, Okumura K, Kumagai Y and Nakano H: Critical contribution of oxidative stress to TNF $\alpha$-induced necroptosis downstream of RIPK1 activation. Biochem Biophys Res Commun 436: 212-216, 2013.

14. He S, Wang L, Miao L, Wang T, Du F, Zhao L and Wang X: Receptor interacting protein kinase-3 determines cellular necrotic response to TNF-alpha. Cell 137: 1100-1111, 2009.

15. Ni Y, Gu WW, Liu ZH, Zhu YM, Rong JG, Kent TA, Li M, Qiao SG, An JZ and Zhang HL: RIP1K contributes to neuronal and astrocytic cell death in ischemic stroke via activating autophagic-lysosomal pathway. Neuroscience 371: 60-74, 2018.

16. Deng Y, Yao L, Chau L, Ng SS, Peng X, Liu X, Au W-S, Wang J, Li F, Ji S, et al: N-Myc downstream-regulated gene 2 (NDRG2) inhibits glioblastoma cell proliferation. Int J Cancer 106: 342-347, 2003.

17. Shen L, Qu X, Li H, Xu C, Wei M, Wang Q, Ru Y, Liu B, Xu Y, Li K, et al: NDRG2 facilitates colorectal cancer differentiation through the regulation of Skp2-p21/p27 axis. Oncogene 37: 1759-1774, 2018 .

18. Mitchelmore C, Büchmann-Møller S, Rask L, West MJ, Troncoso JC and Jensen NA: NDRG2: A novel Alzheimer's disease associated protein. Neurobiol Dis 16: 48-58, 2004.

19. Takahashi K, Yamada M, Ohata H, Momose K, Higuchi T, Honda $\mathrm{K}$ and Yamada M: Expression of Ndrg2 in the rat frontal cortex after antidepressant and electroconvulsive treatment. Int J Neuropsychopharmacol 8: 381-389, 2005.

20. Flügge G, Araya-Callis C, Garea-Rodriguez E, StadelmannNessler C and Fuchs E: NDRG2 as a marker protein for brain astrocytes. Cell Tissue Res 357: 31-41, 2014.

21. Takeichi T, Takarada-Iemata M, Hashida K, Sudo H, Okuda T, Kokame K, Hatano T, Takanashi M, Funabe S, Hattori N, et al: The effect of Ndrg2 expression on astroglial activation. Neurochem Int 59: 21-27, 2011.

22. Zhu H, Zhao J, Zhou W, Li H, Zhou R, Zhang L, Zhao H, Cao J, Zhu X, Hu H, et al: Ndrg2 regulates vertebral specification in differentiating somites. Dev Biol 369: 308-318, 2012.

23. Hao FL, Han XF, Wang XL, Zhao ZR, Guo AH, Lu XJ and Zhao XF: The neurovascular protective effect of alogliptin in murine MCAO model and brain endothelial cells. Biomed Pharmacother 109: 181-187, 2019. 
24. Alamri FF, Shoyaib AA, Biggers A, Jayaraman S, Guindon J and Karamyan VT: Applicability of the grip strength and automated von Frey tactile sensitivity tests in the mouse photothrombotic model of stroke. Behav Brain Res 336: 250-255, 2018.

25. Xu M, Yang L, Hong LZ, Zhao XY and Zhang HL: Direct protection of neurons and astrocytes by matrine via inhibition of the NF-кB signaling pathway contributes to neuroprotection against focal cerebral ischemia. Brain Res 1454: 48-64, 2012.

26. Qin AP, Liu CF, Qin YY, Hong LZ, Xu M, Yang L, Liu J, Qin ZH and Zhang HL: Autophagy was activated in injured astrocytes and mildly decreased cell survival following glucose and oxygen deprivation and focal cerebral ischemia. Autophagy 6: 738-753, 2010.

27. Livak KJ and Schmittgen TD: Analysis of relative gene expression data using real-time quantitative PCR and the 2(-Delta Delta C(T)) Method. Methods 25: 402-408, 2001.

28. Smith R, Owen LA, Trem DJ, Wong JS, Whangbo JS, Golub TR and Lessnick SL: Expression profiling of EWS/FLI identifies NKX2.2 as a critical target gene in Ewing's sarcoma. Cancer Cell 9: 405-416, 2006.

29. Li Y, Yin A, Sun X, Zhang M, Zhang J, Wang P, Xie R, Li W, Fan Z, Zhu Y, et al: Deficiency of tumor suppressor NDRG2 leads to attention deficit and hyperactive behavior. J Clin Invest 127: 4270-4284, 2017

30. Takarada-Iemata M, Yoshikawa A,Ta HM, Okitani N,NishiuchiT, Aida Y, Kamide T, Hattori T, Ishii H, Tamatani T, et al: N-myc downstream-regulated gene 2 protects blood-brain barrier integrity following cerebral ischemia. Glia 66: 1432-1446, 2018.

31. Degterev A, Huang Z, Boyce M, Li Y, Jagtap P, Mizushima N, Cuny GD, Mitchison TJ, Moskowitz MA and Yuan J: Chemical inhibitor of nonapoptotic cell death with therapeutic potential for ischemic brain injury. Nat Chem Biol 1: 112-119, 2005.
32. Smith CC and Yellon DM: Necroptosis, necrostatins and tissue injury. J Cell Mol Med 15: 1797-1806, 2011.

33. Chu D, Zhang Z, Li Y, Wu L, Zhang J, Wang W and Zhang J: Prediction of colorectal cancer relapse and prognosis by tissue mRNA levels of NDRG2. Mol Cancer Ther 10: 47-56, 2011.

34. Shi H, Jin H, Chu D, Wang W, Zhang J, Chen C, Xu C, Fan D and Yao L: Suppression of N-myc downstream-regulated gene 2 is associated with induction of Myc in colorectal cancer and correlates closely with differentiation. Biol Pharm Bull 32: 968-975, 2009.

35. Holler N, Zaru R, Micheau O, Thome M, Attinger A, Valitutti S, Bodmer JL, Schneider P, Seed B and Tschopp J: Fas triggers an alternative, caspase-8-independent cell death pathway using the kinase RIP as effector molecule. Nat Immunol 1: 489-495, 2000.

36. Degterev A, Hitomi J, Germscheid M, Ch'en IL, Korkina O, Teng X, Abbott D, Cuny GD, Yuan C, Wagner G, et al: Identification of RIP1 kinase as a specific cellular target of necrostatins. Nat Chem Biol 4: 313-321, 2008.

37. King MD, Whitaker-Lea WA, Campbell JM, Alleyne CH Jr and Dhandapani KM: Necrostatin-1 reduces neurovascular injury after intracerebral hemorrhage. Int J Cell Biol 2014: 495817, 2014.

38. Xu X, Chua KW, Chua CC, Liu CF, Hamdy RC and Chua BH: Synergistic protective effects of humanin and necrostatin-1 on hypoxia and ischemia/reperfusion injury. Brain Res 1355: 189-194, 2010.

This work is licensed under a Creative Commons Attribution-NonCommercial-NoDerivatives 4.0 International (CC BY-NC-ND 4.0) License. 